Horizons philosophiques

\title{
Globalisation et philosophie : notes sur Le palais de cristal
}

\section{Manola Antonioli}

Volume 17, numéro 2, printemps 2007

La pensée de Peter Sloterdijk

URI : https://id.erudit.org/iderudit/802640ar

DOI : https://doi.org/10.7202/802640ar

Aller au sommaire du numéro

\section{Éditeur(s)}

Collège Édouard-Montpetit

\section{ISSN}

1181-9227 (imprimé)

1920-2954 (numérique)

Découvrir la revue

Citer ce compte rendu

Antonioli, M. (2007). Compte rendu de [Globalisation et philosophie : notes sur Le palais de cristal]. Horizons philosophiques, 17(2), 133-151.

https://doi.org/10.7202/802640ar d'utilisation que vous pouvez consulter en ligne.

https://apropos.erudit.org/fr/usagers/politique-dutilisation/ 


\title{
NOTE DE LECTURE
}

\section{Globalisation et philosophie : notes sur Le palais de cristal}

\author{
"Le sujet et l'objet donnent une mauvaise approximation de \\ la pensée. Penser n'est ni un fil tendu entre un sujet et un \\ objet, ni une révolution de l'un autour de l'autre. Penser se \\ fait plutôt dans le rapport du territoire et de la terre." \\ Gilles Deleuze et Félix Guattari, \\ Qu'est-ce que la philosophie?
}

L'ouvrage de Peter Sloterdijk Le palais de cristal, récemment traduit en français ${ }^{1}$, répond au projet très ambitieux de récapituler l'histoire de la globalisation terrestre, dans le but de "fournir, avec les moyens d'un grand réçit d'inspiration philosophique, les contours d'une théorie du temps présent ${ }^{2}$. Cette entreprise, que l'auteur qualifie d'intempestive ou d'impossible, présente à nos yeux un double intérêt philosophique :

1. Tout d'abord, en affirmant clairement la volonté d'écrire un récit philosophique de la globalisation, Sloterdijk vise à soustraire ce thème au monopole des politologues, des sociologues et des journalistes, qui introduisent subrepticement dans les débats contemporains des termes philosophiques non reconnus et non explicités, dont l'usage impropre mène à des distorsions de sens et à de dangereuses constructions mythiques. La globalisation est ainsi souvent présentée comme une prétendue "nouveauté" apparue dans le monde contemporain, d'ordre exclusivement économique, sociologique et politique et sans aucun lien avec la philosophie et son histoire. Dans cet essai dense et argumenté, Sloterdijk veut au contraire démontrer que les différentes formes de globalisation qui se sont succédé dans l'histoire de la théorie et de la pratique du monde occidental en constituent un fondement philosophique essentiel. Ignorer cette dimension essentielle de la globalisation signifie se condamner à ne pas comprendre dans toute leur complexité les phénomènes politiques, économiques, sociaux et culturels qui caractérisent le temps présent au niveau planétaire.

2. En deuxième lieu, ce projet s'inscrit explicitement dans une perspective qu'on pourrait définir, pour utiliser le langage de Deleuze et Guattari (auteurs auxquels Sloterdijk se réfère tout au long de l'ouvrage) 
géophilosophique ${ }^{3}$. La géophilosophie n'est pas une pure et simple négation de l'histoire, mais une perspective qui part du constat de l'impossibilité d'un récit historique unique et de l'extraordinaire pluralité des histoires réelles, qui demande entre autres des principes d'explication d'ordre spatial. Ainsi, pour Deleuze et Guattari, «la philosophie est une géo-philosophie, exactement comme l'histoire est une géo-histoire du point de vue de Braudel4». Si l'historicisme fait agir la nécessité à travers l'élément historique, y compris dans le destin qui préside au développement du concept, la géographie fait au contraire valoir plutôt la contingence, préfère le devenir à l'histoire, affirme la puissance des milieux, des ambiances, des territoires, des frontières, des lignes de partage mouvantes.

Dans cette approche "spatialisée» et "spatialisante» de la pensée, Sloterdijk affirme, dès le premier chapitre de l'ouvrage ("Des grands récits"), que depuis que l'ère du monopole de l'«Histoire" ou "d'une adulation unilatérale du temps" semble révolue, l'espace réclame de rentrer dans ses droits. Se réclamant de Kant, qui était déjà conscient dans Qu'est-ce que s'orienter dans la pensée? que la raison elle-même prend modèle sur l'orientation dans l'espace, Sloterdijk définit la philosophie comme "son lieu saisi en pensées".

\section{Trois globalisations}

Sloterdijk réfléchit sur la figure du globe comme "un bâtard géographico-philosophique». La question "globalisée» du site terrestre n'a cessé de se déployer au fil de la modernisation. Dans la cosmologie de l'Antiquité occidentale, sous sa forme grecque et hellénistique, la thèse du cosmos visait à représenter le tout de l'Étant à travers l'image d'une sphère regroupant toute chose; la première globalisation a été réalisée par l'ontologie classique comme sphérologie.

La deuxième globalisation, définie par l'auteur comme une globalisation terrestre, a pris forme concrètement entre 1492 et 1945 comme l'époque à laquelle le système actuel du monde a pris ses contours, époque accomplie par la navigation christiano-capitaliste et le colonialisme des États-nations européens. Si les Anciens mesuraient un globe idéalisé avec des lignes et des intersections, les Modernes ont parcouru avec des navires un globe réel. L'époque contemporaine a introduit de nouveaux médias symboliques et techniques, en faisant circuler des avions et des signaux électroniques autour de l'atmosphère jusqu'à déspatialiser progressivement le globe réel et à installer, «à la place du globe terrestre 
courbé, un point presque dépourvu d'extension ou un réseau de points d'intersections et de lignes qui ne représentent que des ordinateurs situés à n'importe quelle distance les uns des autres ${ }^{5}$ ". La disparition progressive du sentiment d'étendue qui caractérisait les Temps modernes produit aujourd'hui un malaise diffus dont il faut s'efforcer de comprendre les causes.

Pour Sloterdijk, la globalisation terrestre commencée avec la modernité européenne et qui est en train de s'achever aujourd'hui n'est pas une histoire parmi d'autres, mais la seule séquence événementielle qui peut porter le nom d' "histoire" ou d' «histoire du monde" dans un sens philosophiquement pertinent, comme affirmation unilatérale des nations européennes en expansion sur la planète, comme conception d'un espace homogène, d'un temps homogène et de l'argent comme valeur universelle.

\section{Les géographes et les marins, la carte et l'océan}

Dans les Temps modernes, ce ne sont plus les métaphysiciens, mais les géographes et les marins qui sont chargés de dessiner la nouvelle image du monde. La globalisation terrestre a mis en œuvre une "catastrophe des ontologies locales", une progressive déterritorialisation généralisée, puisque tous les pays de la vieille Europe ont fini par devenir des "sites" sur une surface globalisée. Sloterdijk définit le site comme un point perçu de l'extérieur, comme une ancienne patrie qui s'offre au regard désenchanté des explorateurs qui reviennent de leurs voyages et de leurs découvertes.

Les temps modernes annoncent ainsi un nouveau message topologique, évangile ou dysangile, bonne et mauvaise nouvelle en même temps : «les gens sont des créatures vivantes qui existent en marge d'un corps rond irrégulier - un corps qui, en tant que tout, n'est pas un utérus, ni un vase, et n'a pas d'abri à offrir où l'on serait en sécurité6».

Cette déterritorialisation s'est accompagnée également du changement de l'élément dominant dans l'image du monde moderne : au XVle siècle, on découvre en effet la prépondérance des surfaces aqueuses sur la planète Terre. La globalisation nautique a contribué à libérer les Européens de leurs anciens ancrages sphériques et locaux.

L'élément aquatique est un espace lisse qui est soumis à un "striage» de plus en plus efficace au cours de l'histoire. J'emprunte cette distinction entre des espaces lisses et des espaces striés à Deleuze et Guattari7; qui 
à leur tour (dans Mille plateaux) la trouvent chez Pierre Boulez, qui s'en servait pour différencier deux espaces-temps musicaux : dans l'espace strié la mesure peut être régulière ou irrégulière, mais elle est toujours assignable, tandis que dans l'espace lisse on peut réaliser des coupures ou des écarts où l'on veut. Ces deux dimensions de l'espace ne s'opposent pas simplement, mais elles n'existent que par leurs mélanges : l'espace lisse peut être strié, alors que dans l'espace strié une dimension lisse et nomade peut toujours refaire surface.

Dans Mille plateaux, Deleuze et Guattari (à partir d'une lecture de Paul Virilio) montrent que la mer a été toujours historiquement un exemple particulièrement complexe des lignes de partage incertaines entre le lisse et le strié. Elle a été d'abord un espace lisse et menaçant où toute orientation était impossible, une sorte de désert liquide. La navigation a mis des siècles à abandonner les côtes; naviguer a signifié pendant très longtemps suivre le littoral en évitant le large et ses dangers de mort. Les navires ne perdaient de vue les côtes qu'exceptionnellement, quand la mer les emportait au large ou quand ils empruntaient quelques parcours reconnus et balisés. Les Portugais, au début du XVe siècle, eurent beaucoup de difficultés à maîtriser les obstacles de la navigation hauturière dans l'Atlantique, qui était à l'époque une nouveauté absolue. Cependant, au fil des siècles et des progrès de la navigation, la mer a fini par devenir le paradigme même d'un "striage» de l'espace à des fins stratégiques, militaires et commerciales. À partir d'une navigation qui était à l'origine empirique et incertaine, l'espace maritime a été progressivement strié en fonction de l'acquisition astronomique du point (obtenu par des calculs à partir d'une observation exacte des astres et du soleil) et de l'acquisition géographique de la carte (qui entrecroise les longitudes et les latitudes jusqu'à quadriller toute la surface du globe).

Sloterdijk évoque pour sa part les "adieux à l'Est»8 : avec les départs en direction de l'Ouest, les Européens ont pu commencer à géométriser leurs comportements dans un espace topologique globalisé et à faire disparaître les terrae incognitae présentes autrefois sur les cartes du monde. Qu'il s'agisse du voyage de Colomb en 1492 ou de la pénétration du continent nord-américain au XIXe siècle, les voyages vers l'Ouest ont donné l'impulsion d'une ouverture de l'espace et des trafics pendulaires réguliers entre n'importe quel point des zones explorées.

Avec le caractère routinier et l'optimisation des techniques maritimes, le voyage en mer a progressivement perdu une bonne partie de ses 
mystères; plus tard, la circulation sur rails et les transports aériens ont permis un contrôle presque parfait sur les mouvements réversibles dans des espaces striés.

\section{Les cartes, la religion, les langues}

Après l'épopée des marins aventuriers, les voyages de découverte prennent la forme d'expéditions qui visent à transformer l'inconnu en connu, et notamment à recenser les lieux de la Terre qui n'avaient pas encore fait auparavant l'objet de voyages, de reproductions, de descriptions et d'exploitations. La "découverte» désigne l'acte de trouver quelque chose et la chose trouvée, tout autant que les moyens techniques et symboliques qui ont permis la découverte.

Dans ce contexte, la cartographie a joué un rôle essentiel dans la globalisation; archives et témoignages des découvertes, les cartes ont permis d'affirmer le droit culturel, historique, juridique et politique des découvreurs sur les nouveaux territoires et leurs habitants : "Les cartes sont par conséquent - notamment dans les premiers temps de l'histoire des découvertes - les témoignages immédiats des prétentions de la civilisation à la souveraineté9». La carte ne se limite jamais à reproduire une réalité physique préexistante; elle naît du besoin de localiser, mais elle dépasse toujours la stricte localisation pour inventer de nouvelles frontières et des lignes de partage, pour réaliser le décompte des richesses ou montrer la puissance économique et militaire des territoires qu'elle décrit. En prétendant être la représentation scientifique, objective et fidèle d'un territoire, elle établit artificiellement des continuités géographiques, politiques, économiques et culturelles, intervient pour légitimer des conquêtes militaires et des zones d'influence politique et économique.

Le facteur religieux était aussi omniprésent dans la navigation des premiers temps, et a transformé les hommes des espaces extérieurs en auditeurs du message chrétien, puissant levier du pouvoir colonial européen.

Mais l'époque des grandes découvertes a permis également aux linguistes et aux ethnologues d'étudier une multitude de nouvelles langues étrangères. Ces rencontres ont donné lieu à deux stratégies de pouvoir principales, souvent coexistantes :

1. Imposer la langue des maîtres coloniaux comme langue d'usage universel (stratégie réussie très souvent par l'anglais, l'espagnol et le français). 
2. Traduire dans les langues des colonisés les discours des nouveaux maîtres, pour leur assurer une plus grande capacité de pénétration dans les cultures locales.

La traduction a été ainsi un instrument essentiel de pouvoir; en général les langages européens ont réussi à recouvrir les langues locales, alors que ces dernières n'ont pu que très rarement absorber les idiomes des colonisateurs. Sloterdijk n'évoque pas, cependant, tous les phénomènes de créolisation à travers lesquels les langues "dominantes" ont été modifiées en profondeur par les langues "dominées", jusqu'à produire des composés linguistiques imprévisibles et inédits ${ }^{10}$.

\section{Un monde synchronisé}

Dans la troisième globalisation, celle que nous vivons actuellement, la terre se présente selon Sloterdijk comme un corps entouré d'écorces virtuelles, qui ont remplacé le ciel éthéré. La fluidification omniprésente a fait de toutes les villes de la planète des villes portuaires, où l'information forme des courants dans des océans de données.

Avec les progrès de la mise en réseau du monde, qui est le présupposé essentiel pour fonder une théorie de l'ère contemporaine, se développent les symptômes d'une misanthropie universelle, comme réaction violente à des voisinages réels ou à distance instaurés entre les hommes. La "culture" et le "cosmopolitisme" ne disparaissent pas pour autant, mais se présentent désormais comme des mesures destinées à atténuer la misanthropie. S'annonce ainsi une vraie révolution anthropologique, qui nous oblige à compter en permanence avec l'autre éloigné, perçu souvent comme étranger ou ennemi.

Dans les Temps modernes, les voyages unilatéraux des Européens vers les Autres ont permis d'explorer l'horizon anthropologique des peuples et des cultures de la planète. "L'humanité" a pu ainsi se concrétiser réellement, comme le dernier épisode de cette «histoire universelle». Depuis peu, ce concept a acquis une nouvelle complexité et une profondeur insoupçonnée auparavant, au fur et à mesure qu'il prend forme également dans le sens inverse, à travers le regard porté par les Autres sur les Occidentaux.

Comme l'«histoire universelle" dont Deleuze et Guattari ont retracé les étapes dans L'Anti-CEdipe et Mille plateaux, celle qu'évoque Sloterdijk est une «histoire universelle du fortuit», le résultat des devenirs imprévisibles de la contingence et de l'immanence. 
Le "dernier globe» n'admet plus aucune construction verticale ou totalisante (supermonosphère ou centre de tous les centres) mais ne permet de vastes constructions qu'à l'horizontale, à travers des assemblages, des montages intersubjectifs et interculturels, selon un modèle très proche de celui décrit par Deleuze et Guattari comme le devenir rhizomatique de multiples "agencements collectifs d'énonciation", qui doivent encore être produits et inventés pour que nous puissions de nouveau naviguer sur les océans inconnus qui s'ouvrent à nous.

\section{Le soi sans lieu et le lieu sans soi}

Sloterdijk résume les constats qui reviennent régulièrement dans la littérature actuelle sur la globalisation :

1. Il faut négocier de nouvelles formes d'organisation à des échelles intermédiaires entre le local et le global.

2. Les communautés politiques sont de plus en plus situées au-delà de l'État-nation.

3. Le monde globalisé voit s'accroître le fossé entre pauvres et riches.

4. Les problèmes environnementaux transformeront nécessairement l'humanité en une communauté (même forcée) d'intérêts écologiques.

Tous ces thèmes reconnaissent la tendance à la dissipation des conceptions traditionnelles sur les sujets et les unités au niveau social et politique, ce qui induit (dans le langage de Sloterdijk) la nécessité "d'une poétique politique de l'espace ou d'une "macrosphérologie"11". La crise de l'État nation signifie la fin d'une structure domestique et immunitaire, réelle et imaginaire à la fois, qui permettait autrefois la convergence du lieu et du soi et la création d'une identité régionale protégée. On sait désormais que ce lien entre le lieu et le soi est le fruit d'une invention et d'une construction et qu'il n'a rien de stable et de "naturel». Déjà en 1993, dans son "essai sur l'hyperpolitique" intitulé Dans le même bateau, Sloterdijk avait analysé le malaise affectant toute la civilisation mondiale comme une crise de la forme politique du monde, comme un symptôme lié au passage de «l'esprit de l'ère agraire" caractéristique de la politique classique vers le nouveau jeu mondial de l'ère industrielle, dont on n'a pas encore réussi à établir les règles :

Dans la mesure où la politique, dans sa structure classique, a représenté l'art de l'appartenance dans les villes et les grands empires de l'époque agraire, la "mort de Dieu» annonce pour elle un mouvement critique. Les concepts spatiaux qui étaient à l'œuvre dans cette période intermédiaire marquée par le 
travail du sol deviennent caduques face à un univers synchrone dont les effets sont de plus en plus évidents. Les acteurs du nouveau jeu mondial de l'ère industrielle ne se définissent plus par rapport au sol et à la "patrie» mais par des accès aux gares, aux terminaux et à toutes sortes de possibilités de raccordement. Pour eux le monde est une hyperbulle câblée ${ }^{12}$.

Quand on desserre l'entrelacs entre les lieux et les formes du soi, on aboutit selon Sloterdijk à deux positions extrêmes, celle d'un soi sans lieu et celle d'un lieu sans soi. Toutes les sociétés ont cherché dans le cours de leur histoire à réaliser des compromis entre ces deux pôles extrêmes, et dans le futur toute communauté politique devra également répondre au double impératif de la détermination de soi et du lieu.

La figure la plus proche du Soi sans lieu a été le judaïsme de la diaspora, un peuple sans pays qui n'avait que le Livre comme patrie. Dans sa période d'exil, le judaïsme a brandi constamment sous les yeux des peuples sédentaires le "scandale» d'un soi-peuple existant sans lieu, en dehors d'un territoire comme conteneur et d'un enracinement dans le sol. Les groupes sédentaires ont toujours, bien au contraire, conçu le sol qui les porte comme la.garantie unique de leur immunité symbolique et de leur cohésion; cette illusion identitaire est toujours dangereuse, puisqu'elle détermine tous les réflexes violents de "défense du pays", dont ont été également victimes (avec les conséquences désastreuses que l'on connaît) les citoyens d'Israël après 1948.

Cet héritage historique de l'ère sédentaire apparaît de moins en moins légitime, au fur et à mesure que la mobilité transnationale relativise les liens entre les peuples et les lieux et qu'on assiste à la création de "communautés imaginaires» qui existent en dehors des États-nations.

Le lieu sans soi prend forme dans les régions inhabitables de la Terre (monde polaire, hautes montagnes, forêts vierges, déserts de sable et océans), qui sont d'ailleurs de plus en plus menacés de disparition suite aux effets de l'action humaine. II faut également leur adjoindre tous les déserts artificiels produits par l'homme : lieux de transit (gares, ports, aéroports, autoroutes), centres commerciaux ou villages de vacances, liste à laquelle il faudrait ajouter les banlieues des grandes métropoles ${ }^{13}$. II s'agit de lieux avec lesquels aucune relation d'habitat, de séjour, de demeure n'est plus possible pour les hommes qui les fréquentent, ce qui produit une sensation de malaise diffus et généralisé. 
La crise des sociétés contemporaines dérive ainsi de l'érosion progressive du lien du soi avec un territoire, étayé par des symboles partagés et situé dans un contexte monoculturel et monolingue; le collectif national se construisait dans un hermétisme territorial à caractère immunitaire, mis désormais définitivement en cause par la globalisation ${ }^{14}$.

La globalisation s'accompagne ainsi nécessairement de contestations : "La contestation de la globalisation est aussi la globalisation elle-même - elle fait partie de la réaction immunitaire et indispensable des organes locaux aux infections provoquées par l'agrandissement du format du monde ${ }^{15}$ ".

Les postmodernes s'efforcent ainsi de construire de nouvelles situations d'immunité viables, qu'ils trouvent (selon Sloterdijk) dans les formes de vie individualistes qui caractérisent aujourd'hui les sociétés avancées, où les individus tendent à se séparer des groupes et de l'être-ensemble de la communauté politique, perçus autrefois comme protecteurs. Cette tendance s'exprime avec le plus de clarté aux États-Unis, où les gens pratiquent "le souci de soi biologique, psychotechnique et religioïde, parallèlement à une abstinence croissante d'engagement politique ${ }^{16}$ ». Les individus individualisés ont renoncé à exprimer le monde, à devenir des "grands hommes" ou des hommes microcosmiques, et Sloterdijk semble présenter cette tendance comme irréversible et destinée à se généraliser, en tant que stade final de la culture.

On pourrait au contraire affirmer (en pariant sur l'avenir politique des humanités qui peuplent le globe) que l'existence de ces mécanismes de réaction immunitaire et auto-immunitaire qui poussent à l'isolement individualiste, à l'hédonisme tragique du consommateur et de l'habitant des métropoles, quoique incontestable, n'est en rien la dernière possibilité d'existence qui s'ouvre à nous dans le contexte de la troisième globalisation.

Si l'État-nation et les politiques qu'il inspirait sont en perte de vitesse et si aucune doctrine ou parti politique ne peut plus prétendre donner voix à la totalité des aspirations d'un peuple et d'une nation, le politique survit à ces mutations et ne cesse de se manifester sous de nouvelles formes. En témoigne aujourd'hui (malgré les critiques qu'on peut adresser à ce concept et à son instrumentalisation médiatique), l'intérêt pour de nouvelles perspectives de "démocratie participative" qui pourraient dans le futur assurer une participation accrue des citoyens aux grandes orientations politiques, économiques et scientifiques collectives, mais 
aussi les multiples formes d'activisme politique non-étatique, à travers lesquelles des individus et des groupes aspirent à faire de la politique sans viser le pouvoir et le gouvernement, sans vouloir incarner le destin historique d'un peuple et d'une nation, toutes les formes d'association transversale et horizontale à propos desquelles Deleuze et Guattari parlaient de «politique mineure».

Sloterdijk lui-même évoque d'ailleurs dans ce même ouvrage la nécessité de concevoir de vastes constructions horizontales, ce qui n'exclut pas à ses yeux quelques constructions élevées : dans cette perspective, l'«individu individualisé» et le citoyen transformé en consommateur ne sont probablement pas - il faut au moins le souhaiter - le stade final de la culture. La pensée de Sloterdjik offre de nombreux outils conceptuels en vue d'une nouvelle interprétation philosophique de l'individu, qu'il développe dans le projet des Sphères ${ }^{17}$ : chaque individu est toujours accordé à travers des rythmes, des mélodies, des projets collectifs avec des millions d'autres, forme une dyade avec un lieu, est niché dans une sphère, un globe ou une écume dont les dimensions et les connexions se modifient dans le cours de l'histoire individuelle et collective :

À chaque forme sociale s'attache une maison du monde spécifique, une cloche de sens sous laquelle des créatures humaines commencent par se collecter, se comprendre, se défendre, s'exacerber, sortir de leurs frontières. Les hordes, les tribus et les peuples, et plus encore les empires, sont, dans leurs formats respectifs, des entités psycho-sociosphérique(s) qui s'aménagent, se climatisent, se contiennent elles-mêmes. À chaque instant de leur existence, ces entités sont forcées de placer au-dessus d'elles, avec leurs moyens typiques, leur propre ciel sémiotique à partir duquel leurs inspirations communes, qui constituent leur caractère, s'écoulent vers elles ${ }^{18}$.

Les individus insulaires engagés dans les processus postmodernes de globalisation sont affectés par une "pathologie des sphères", qui les entraîne dans un processus d'écumisation, qui métamorphose en profondeur les créations de contextes et les architectures de l'immunité, et qui ne peut être étudié(e) qu'à l'aide d'une «amorphologie politique».

La métaphore architecturale choisie par Sloterdijk pour représenter l'espace protégé du monde capitaliste avancé, est le Crystal-Palace, bâtiment aux dimensions symboliques incommensurables inauguré à Londres en 
1851 dans le cadre de la première exposition mondiale ${ }^{19}$, et détruit en 1936 par un incendie. II s'agissait à l'époque d'un vrai prodige technologique qui inaugurait une nouvelle esthétique de l'immersion qui préfigure l'évolution du monde contemporain avancé vers un espace conçu comme un grand intérieur protégé. Le palais de cristal visait à transposer le monde extérieur en tant que tout dans un espace circonscrit, transfiguré par le luxe et la transparence. Image spatialisée de la «fin de l'Histoire», cette invention architecturale aspire à intégrer la nature et la culture dans un intérieur élargi, dans un espace interne doté de tous les conforts et d'un climat artificiel; la vie sociale commence ainsi à être conçue comme un habitat protecteur, destiné à protéger les ex-citoyens devenus désormais consommateurs des événements historiques, des conflits et des aléas du monde extérieur.

Le monde occidental est pour Sloterdijk incarné aujourd'hui dans un grand intérieur de ce type, une serre de la détente dédiée au culte joyeux du consumérisme 20 . Ce bâtiment prophétique révèle ainsi que le capitalisme a toujours été et voulu être autre chose qu'un simple rapport de production; il s'agit plutôt d'un projet global qui consiste à transposer la totalité de la vie, du travail, des désirs et de l'expression des êtres dans l'immanence du pouvoir d'achat.

Le royaume actuel du pouvoir d'achat se concrétise dans le cadre d'une réalité indoors généralisée, dans le palais capitaliste du monde décrit aussi récemment par Negri et Hardt dans Empire ${ }^{21}$. À ce propos il faut remarquer que, tout en reconnaissant à ces deux auteurs le mérite d'avoir traité philosophiquement la question de la globalisation, Sloterdijk ne se confronte jamais directement à leurs thèses, se limitant à les définir comme des "marxistes ultratardifs»22 ou, dans Écumes, en soulignant (d'ailleurs à juste titre) la dimension religieuse de l'ouvrage, qui s'inscrit dans la tradition de la théologie chrétienne de l'histoire ${ }^{23}$. Une discussion plus approfondie des thèses exposées dans leur ouvrage aurait pourtant permis à Sloterdijk de mieux expliciter son point de vue sur la réalité concrète des dimensions économiques et des perspectives politiques de la troisième globalisation, alors qu'il se concentre pour l'essentiel sur ses composantes civilisationnelles, culturelles et sociologiques et qu'il n'envisage que très rarement les sorties possibles de ce cauchemar climatisé.

Sloterdijk reconnaît cependant que ce palais n'a pas vocation à inclure toute l'humanité : cette grande structure intègre de nouveaux habitants 
qui en étaient autrefois exclus (il suffit de penser aux pays de l'Europe de l'Est, aux pays comme la Chine et l'Inde qui se développent très rapidement), mais elle repousse également d'anciens membres et menace d'exclusion ceux qui y sont géographiquement inclus mais qui sont menacés de perte du pouvoir d'achat. Cette image est donc déjà inadéquate à décrire les temps présents, puisqu'elle semble présupposer une frontière bien définie entre l'intérieur capitaliste et son extérieur; nous savons effectivement (Deleuze et Guattari, Negri et Hardt l'ont largement montré) que les frontières de cette construction sont poreuses et incertaines. Il y a des zones de pauvreté et de misère de plus en plus vastes dans les enclaves de prospérité et, en même temps, des zones de richesse ne cessent d'apparaître dans les pays les plus pauvres; les limites entre centre et périphérie, Nord et Sud du monde sont en transformation constante.

Si le palais de cristal n'inclut pour l'instant qu'un tiers de l'humanité, c'est parce qu'il est impossible d'organiser matériellement une intégration de tous les membres du genre humain dans les conditions actuelles de la technique, des ressources énergétiques et de l'économie. La persistance de ce modèle illusoirement protégé, caractérisé par la richesse, le consumérisme et le gaspillage des ressources naturelles, n'est en effet concrètement possible que grâce à la persistance de zones internes et externes de pauvreté et de sous-développement qui constituent des réserves indispensables de matières premières et de main-d'œuvre à bas prix pour les habitants du palais de cristal. Le développement économique actuel de la Chine, par exemple, n'a été possible que par l'exploitation des travailleurs pauvres en provenance des régions rurales et arriérées du pays.

Même si Sloterdijk ne consacre pas beaucoup d'analyses à la dureté économique et sociale des règles de fonctionnement du monde qui permettent la survie de ce modèle de société, il reconnaît clairement que derrière l'idéologie de la globalisation comme réalité universelle se cache l'existence d'un continent artificiel qui flotte sur un océan de pauvreté : «L'exclusivité est inhérente au projet palais de cristal en tant que tel24».

Les débats sur la globalisation prennent ainsi souvent la forme d'un monologue des zones de prospérité, alors que la majorité des autres régions du monde ne connaissent souvent que les effets secondaires défavorables de ces phénomènes et essaient plutôt de les contrecarrer (à travers des tentatives d'organisation économique Sud-Sud comme en Amérique Latine ou dans le cadre des forums altermondialistes). 


\section{Surabondance et décroissance}

Sloterdijk constate un renversement profond de la pensée traditionnelle, axée sur les notions de pénurie, d'urgence et de manque; les populations de la sphère du confort s'orientent actuellement plutôt vers une existence faite d'options, qu'il désigne comme une existence de "gâterie" et de "surabondance». Pour vaincre l'ennui fondamental qui caractérise la vie dans la grande serre, apparaît une agitation constante de décharge généralisée, de poursuite de caprices individuels et de goûts personnels. La surabondance à l'intérieur du palais de cristal multiplie les facilités d'accès à tout ce qui existe sous forme de marchandises, facilité due aussi aux systèmes de transports modernes fondés sur le pétrole; dans ce contexte, une forme plus légère de la subjectivité (le "Soi usager») commence à remplacer la forme plus lourde des Temps modernes (le «Soi cultivé»).

C'est l'introduction des énergies fossiles qui a permis la référence au principe surabondance dans la civilisation du confort, et pour Sloterdijk la course à la surabondance restera la caractéristique prégnante des situations futures, même si le cycle de l'énergie fossile arrive à son terme. Les combustibles renouvelables et les technologies solaires devraient en effet permettre l'essor d'une ère post-fossile qui permettra de poursuivre la consommation et le gaspillage, tout en privilégiant plutôt des flux immatériels.

Cette anticipation constitue à mes yeux le point le plus faible et le plus discutable de l'ouvrage de Sloterdijk. S'il est vrai que les sociétés avancées actuelles vivent depuis longtemps dans la surabondance et le gaspillage, Sloterdijk sous-estime ici lourdement la portée des enjeux environnementaux et écologiques qui s'annoncent pour le XXle siècle, et qui ne se résument pas à la fin du pétrole et à l'introduction de nouvelles formes d'énergie.

Le modèle de développement sans bornes que le monde occidental a poursuivi et a cherché à imposer au reste de la planète, est entré dans une crise irréversible puisque nous prenons de plus en plus conscience de notre dépendance à l'égard d'un monde fini qui implique des ressources finies. S'annonce ainsi la nécessité d'un changement de voie pour la technique, la société et les modes de vie au niveau mondial, qui ne permettra pas à l'Occident de poursuivre en direction d'une exploitation effrénée des ressources planétaires. Les modèles exclusifs de la croissance et du développement ont entraîné une catastrophe 
écologique qui nous impose aujourd'hui un contrôle de la croissance et de l'exploitation des ressources naturelles à un niveau planétaire, le développement d'un vaste projet d'écologie politique qui est incompatible avec le modèle de surabondance prônée par les sociétés occidentales ${ }^{25}$.

Ces considérations sur une "ère post-fossile" et les perspectives de consommation infinie qu'elle serait encore susceptible d'assurer dans l'avenir, contrastent par ailleurs avec les positions exprimées par Sloterdijk dans des ouvrages précédents, où il a exploré les fondements philosophiques d'une nouvelle écologie politique. En 1989, dans La mobilisation infinie, il a en effet fourni les éléments d'une «esquisse pour le fondement d'une critique de la cinétique politique", en interprétant I'histoire des Temps modernes comme le lieu d'une "mobilisation infinie», de plus en plus difficile à arrêter, et qui ouvre désormais la voie à une possible auto-extinction de l'humanité : "Voilà qui nous procure la formule des processus de modernisation : le progrès est mouvement vers le mouvement, mouvement vers plus de mouvement, mouvement vers une plus grande aptitude au mouvement26". Sloterdijk prônait ainsi une "démobilisation", qui pourrait constituer aujourd'hui un point de départ philosophique en direction d'une pensée de la "décroissance», telle qu'elle est développée par de nombreux courants de la pensée écologiste. La dernière partie de l'ouvrage laissait en outre émerger la figure de la Terre, devenue dans le cours de l'histoire de la modernité le simple arrière-plan oublié d'un procès-progrès engagé dans la logique absolue de la mobilisation :

L'indifférence du drame pour le lieu où il se déroule est inscrite
dans la logique de la mobilisation. C'est seulement au moment
où la pièce menace de ruiner le plateau qu'une nouvelle
perception de soi-même s'impose aux acteurs, il fallait que
l'humanité mue par l'histoire attende les impérialismes des
temps modernes, l'ère industrielle et la civilisation planétaire
des médias pour que la crise de ses fondements lui montre la
vérité de sa propre entreprise27.

L'émergence d'une pensée de la "durabilité" (sustainability) depuis quelques années nous pousse enfin à constater que le processus industriel à grande échelle continue de détruire davantage de réserves naturelles et humaines qu'il ne peut en produire28. La planète ne peut plus être un simple théâtre pour la mise en place de la pièce jouée par une humanité conquérante, mais un contenu du souci humain : "Par là, toutes 
les prémisses du jeu historique changent. Ce qui a été la scène devient le thème des événements. Ce qui servait d'arrière-plan s'intègre au premier plan. Ce qui était là comme matière première apparaît comme produit. $\mathrm{Ce}$ qui était la scène devient la pièce elle-même ${ }^{29}$ ".

\section{L'incompressible}

Dans le système actuel du monde, tout est placé sous la contrainte du mouvement, du nomadisme et de la mobilisation permanente. La déterritorialisation, qui a été autrefois la conquête essentielle des Temps. modernes, fait partie désormais du quotidien et de sa banalité. La télévision nous donne à voir le spectacle d'un monde débarrassé de ses frontières, les réseaux de communication et la rapidité des transports ont comprimé l'espace, nous donnant l'illusion de sa disparition. Mais on assiste déjà à un mouvement de correction de ces tendances, mouvement qui vise à réévaluer l'espace ignoré; la culture de la présence commence à faire valoir de nouveau ses droits en vue d'une insurrection contre le monde rétréci et d'une redécouverte de la lenteur.

Mais le retour au «local» ne signifie pas nécessairement un retour à une pensée "réactionnaire" de l'espace; il s'agit plutôt d'un retour sur la scène de la dimension asymétrique des lieux. Malgré les progrès de la décontextualisation, de la compression et de la neutralisation de l'espace, ce dernier n'est pas simplement réductible à une distance à parcourir le plus rapidement possible, mais constitue un ensemble discontinu de singularités.

Les discours sur la globalisation sont donc souvent hyperboliques et sous-estiment la persistance et la nécessité d'ancrages dans le local, dans les territoires et la singularité des cultures : "Le localisme n'est pas de nature réactive mais doit être compris comme l'affirmation de l'extension-sur-le-lieu créative ${ }^{30}$ ".

On pourrait reformuler cet "éloge de l'asymétrie» de Sloterdijk, encore une fois, dans le langage de Deleuze et Guattari. Contrairement à ce qu'on affirme souvent, suite à des lectures trop hâtives, déterritorialisation et nomadisme d'une part, territorialisation et sédentarité de l'autre, ne constituent pas dans Mille plateaux deux pôles opposés, dont l'un serait toujours positif et l'autre toujours négatif. II s'agit plutôt de plusieurs lignes, plusieurs plans qui traversent chaque individu et chaque groupe : tout territoire peut s'ouvrir sur son dehors, tout mouvement de déterritorialisation implique un lien avec un territoire. Penser toujours en géographe ou en géophilosophe, signifie aussi comprendre que toute 
pensée dichotomique est inadéquate pour saisir la complexité du monde actuel, où nous sommes submergés par d'innombrables informations et sollicitations qui semblent menacer nos possibilités d'intelligibilité. Nord et Sud, simulacre et réalité, territorialisation et déterritorialisation, enracinement et déracinement sont des catégories qui ne peuvent jamais être simplement opposées si on veut penser notre ère planétaire. La mondialisation entraîne le repli identitaire et communautaire, la désacralisation du monde détermine paradoxalement un retour de nouvelles formes, de plus en plus menaçantes, de conflits religieux, le capitalisme triomphant doit affronter de petites machines de guerre qui l'obligent à modifier ses stratégies, l'explosion du virtuel suscite de nouvelles formes d'expérience et de création qui essaient de compenser la perte d'expérience qui nous menace, le déclin des formes traditionnelles d'art appelle d'autres formes de production esthétique : une logique transversale, ouverte sur la complexité des devenirs, est de plus en plus nécessaire pour lire ces nouvelles cartes aux frontières mouvantes.

Le territoire n'est jamais une donnée «naturelle» et immuable, ni un système clos sans relations avec le dehors, mais il est le fruit d'une invention collective, aux frontières entre le réel et l'imaginaire. Ainsi, il peut aussi devenir aujourd'hui une forme paradoxale de résistance face à l'entreprise de lissage total des espaces et des identités, à la dynamique implacable d'une déterritorialisation unilatérale et sans limites imposée à la planète par le capitalisme avancé. La déterritorialisation ne doit pas être exclusivement un moyen d'effacer la multiplicité des rythmes, des formes de subjectivation individuelles et collectives, des enracinements territoriaux et culturels, mais devenir au contraire un outil de construction de formes de subjectivation qui puissent intégrer tous ces divers éléments dans la découverte de nouvelles voies de singularisation.

\section{Post-historique et post-colonial}

L'ouvrage de Sloterdijk défend aussi une thèse forte et provocatrice sur la «fin de l'histoire», dont il propose une nouvelle version. Pour l'auteur, l'époque de la globalisation est la seule à pouvoir être qualifiée d'histoire, histoire dont le contenu est le drame de l'exploration de la Terre, et qui a abouti à une situation dans laquelle, pour la grande majorité des habitants de la planète, l'image géographique du globe terrestre dit la vérité sur leur situation.

La séquence des événements qui ont eu lieu entre 1492 et 1945 reste la seule qu'on puisse donc caractériser comme historique, si on considère 
que "l'histoire" est le mythe de la naissance du système mondial31". La vitalité de l'histoire et l'intense activité des historiens ne constituent en rien un démenti de cette thèse aux yeux de Sloterdijk : la résistance du métier fera en sorte encore longtemps que des historiens infatigables aient l'illusion de vivre encore dans l'histoire, et les récits historiques ne cesseront pas de jouer une fonction mytho-dynamique active pour les groupes et les institutions :

Rien de tout ce qui constitue une différence entre le ciel et la terre ne peut ainsi échapper aux historiens infatigables. (...) Ils écrivent l'histoire de la menstruation au Moyen Âge; ils écrivent l'histoire des projectiles, depuis le javelot de l'ère glaciaire jusqu'au missiles intercontinentaux; ils écrivent l'histoire du tag et celle du gangsta-rap; l'histoire des dix plus grandes fortunes de la terre; l'histoire de la copie pirate depuis l'ouverture de la Chine; ils écrivent aussi l'histoire de la psychothérapie à orientation corporelle dans le Sauerland, en Rhénanie-Westphalie. Ils écrivent l'histoire des matières plastiques; I'histoire des contributions des intellectuels afro-caraịbéens au discours critique sur l'eurocentrisme; ils écrivent l'histoire de la dégénérescence graisseuse des animaux domestiques aux États-Unis avant le 11 septembre; I'histoire des prix Nobel et celle des succédanés du sucre ${ }^{32}$.

La perspective politique de la période proprement historique a été une perspective unilatérale de domination qui s'est exprimée dans les divers épisodes coloniaux qu'a produits l'expansion européenne sur la planète. La pratique coloniale se fondait sur la croyance inébranlable des "grandes nations" occidentales en leur droit de conquête et de soumission des autres peuples. Aujourd'hui, il faut constater la fin de cette unilatéralité qui a duré plusieurs siècles et reconnaître que plusieurs autres pages s'ouvrent dans la "posthistoire" de la planète : l'exigence d'une lecture symétrique et plurielle du monde s'exprime ainsi depuis longtemps dans les Postcolonial Studies.

Malgré ce constat, Sloterdijk (tout comme Negri et Hardt dans Empire) observe le monde presque exclusivement de l'intérieur (encore essentiellement européen et nordaméricain) du palais de cristal, sans jamais essayer d'élargir la perspective et de multiplier les points de vue à partir du regard que les cultures "non-occidentales" portent sur cet espace et sur son histoire. Si on essaye de prendre au sérieux ce 


\section{Manola Antonioli}

concept, une posthistoire implique de multiplier les mondes et les humanités après la fin de l'unilatéralisme.

Le récit historique et philosophique de la globalisation exposé dans Le palais du cristal appelle un prolongement nécessaire sous la forme d'un récit posthistorique et postcolonial, qui puisse nous permettre d'accéder à de nouvelles régions du monde :

Les certitudes les plus fondées, quand il arrive que la plongée aux certitudes rencontre un fond, disent que nous entrons tous maintenant dans une nouvelle région du monde, qui désigne ses lieux sur toutes les étendues données et imaginables, et dont seuls quelques-uns avaient pu prévoir au loin les errances et les obscurités

\section{Manola Antonioli \\ Collège International de Philosophie, Paris}

1. Peter Sloterdijk, Le palais de cristal. À l'intérieur du capitalisme planétaire, Paris, Maren Sell Éditeurs, 2006.

2. Ibid., p. 11.

3. Au sujet de la perspective géophilosophique de l'œuvre de Deleuze et Guattari, je me permets de renvoyer à mon ouvrage Géophilosophie de Deleuze et Guattari, Paris, L'Harmattan, 2004.

4. Gilles Deleuze et Félix Guattari, Qu'est-ce que la philosophie?, Paris, Minuit, 1991, p. 91.

5. Peter Sloterdijk, Le palais de cristal, op. cit., p. 25.

6. Ibid., p. 49.

7. Gilles Deleuze et Félix Guattari, Mille plateaux, Paris, Minuit, 1980, plateau 14 («1440 Le lisse et le striém).

8. Peter Sloterdijk, Le palais de cristal, op. cit., première partie, chap. 5 («Adieux à l'Est, entrée dans l'espace homogènem).

9. Ibid., p. 154.

10. À ce sujet, je renvoie aux travaux essentiels d'Édouard Glissant.

11. Peter Sloterdijk, Le palais de cristal, op. cit., p. 215.

12. Peter Sloterdijk, Dans le même bateau, Paris, Payot \& Rivages, 1997, p. 57. 
13. C'est l'anthropologue Marc Augé qui, dans un ouvrage devenu classique, a exploré les non-lieux, espaces d'anonymat de plus en plus nombreux dans le monde contemporain (Non-lieux, Paris, Seuil, 1992).

14. Pour une mise en perspective historique détaillée de cette crise, je renvoie encore une fois à Dans le même bateau, op. cit.

15. Peter Sloterdijk, Le palais de cristal, op. cit., p. 219. Malgré ce constat essentiel, Sloterdijk n'explore pas les formes multiples de ces contestations (mouvements altermondialistes, manifestations religieuses, nouveaux nationalismes, etc.); il ne consacre des analyses (qu'on ne pourra pas commenter dans les limites restreintes de cet article) qu'au terrorisme (cf. première partie, chap. 34 «Le monde dense et la désinhibition secondaire : du terrorisme considéré comme un romantisme de l'agression purem).

Une lecture analogue de la globalisation et des réactions qu'elle suscite en termes de processus immunitaires et auto-immunitaires a été développée par Jacques Derrida dans Foi et savoir (Paris, Seuil, 2000). Derrida préfère parler plutôt de mondialisation et analyse tout particulièrement dans ce texte les phénomènes de uretour du religieux".

16. Ibid., p. 220.

17. Peter Sloterdijk, Bulles - Sphères I, Paris, Pauvert, 2002; Globes - Sphères II (trad. fr. à paraître en 2007 chez Maren Sell Éditeurs); Écumes - Sphères III, Paris, Maren Sell Éditeurs, 2005.

18. Peter Sloterdijk, Bulles - Sphères I, , op. cit., p. 64.

19. Le Crystal-Palace fait l'objet de longues analyses dans l'ouvrage de Sloterdijk Écumes - Sphères III, op. cit.

20. Le mode de vie des habitants de cette serre capitaliste a été décrit récemment par le philosophe et sociologue Gilles Lipovetsky dans l'ouvrage Le bonheur paradoxal, Paris, Gallimard, 2006.

21. Michael Hardt et Antonio Negri, Empire, Paris, Exils, 2000.

22. Peter Sloterdijk, Le palais de cristal, op. cit., p. 276.

23. Peter Sloterdijk, Écumes - Sphères III, op. cit., p. 730-733.

24. Ibid., p. 280. Sur les inégalités inhérentes à la globalisation, je renvoie aux travaux de Zygmunt Bauman et notamment aux ouvrages Le coût humain de la mondialisation, Paris, Hachette, 1999 et La vie en miettes, Rodez, Le Rouergue/Chambon, 2003.

25. La bibliographie sur ces questions est désormais extrêmement nourrie; je me limiterai à citer quelques ouvrages : Edgar Morin, L'an I de l'ère écologique, Paris, Tallandier, 2007; Serge Latouche, Le pari de la décroissance, Paris, Fayard, 2006 et Survivre au développement, Paris, Mille et une nuits, 2004.

26. Peter Sloterdjik, La mobilisation infinie, Paris, Christian Bourgois éditeur, 2000, p. 35.

27. Ibid., pp. 301-302.

28. La durabilité devient un objet de réflexion pour Sloterdijk dans les dernières pages de Dans le même bateau, op. cit., p. 85-87.

29. Peter Sloterdijk, La mobilisation infinie, op. cit., p. 304.

30. Peter Sloterdijk, Le palais de cristal, op. cit.

31. Ibid., p 227.

32. Ibid., p 239.

33. Édouard Glissant, Une nouvelle région du monde, Esthétique I, Paris, Gallimard, 2006. 